\title{
Normative ranges of tympanometric measurements in cats
}

\author{
Yusuf Sinan ŞİRIN ${ }^{1}$, Özlem Şengöz ŞİRIN ${ }^{1}$
}

${ }^{1}$ Department of Surgery, Faculty of Veterinary Medicine, Burdur Mehmet Akif Ersoy University, Burdur/TURKEY

\section{Key Words:}

cat

otitis media

static admittance

tympanometry

Received : : 19.10 .2020

Accepted : : 02.12 .2020

Published Online : 30.12 .2020

Article Code : 813073

Correspondence:

Ö. ŞENGÖZZ-ŞIRIN

(sengozozlem@gmail.com)

ORCID:

YS. SIRIN

O. SENGOZ-SIRIN: 0000-0003-1322-7290

\begin{abstract}
Middle ear diseases are common in cats. The aim of this study was to determine the normative tympanometric data of cats. Tympanometry, which provides objective data in the examination of the middle ear in humans, can be used as a supplementary examination, especially in cats with middle ear diseases where the external ear canal is normal. In this study, 30 healthy ears belonging to 15 cats which were classified as ASA-I (American Society of Anesthesiologyist) were determined and evaluated according to equivalent ear canal volume $\left(\mathrm{V}_{\mathrm{ea}}\right)$, static acoustic admittance $\left[\mathrm{Y}_{\mathrm{tm}}\right.$ (compliance)], tympanometric peak pressure (TPP) and gradient values under general anesthesia. Tympanometric measurements obtained from right and left ears were $0.61 \pm 0.12 \mathrm{ml}$ and $0.60 \pm 0.12 \mathrm{ml} \mathrm{V}_{\mathrm{ea}}, 0.31 \pm 0.11$ $\mathrm{ml}$ and $0.31 \pm 0.11 \mathrm{ml} \mathrm{Y}_{\mathrm{tm}},-11.2 \pm 26.9 \mathrm{daPa}$ and $-14.9 \pm 29.2 \mathrm{daPa} \mathrm{TPP}, 0.19 \pm 0.11 \mathrm{ml}$ and $0.24 \pm$ $0.18 \mathrm{ml}$ gradient respectively. Tympanometry as a supplementary examination is an easy to perform test in healthy cats with consistent results. It has the advantages of being completed in a short time, easy evaluation and providing information about middle ear without advanced imaging techniques. Results of this study represents the normative tympanometric measurements of cats which can be helpful distinguishing normal and abnormal conditions. Further studies needed to present exact values of standart tympanometric measurements and to introduce other variables like acustic refleks, wideband and multifrequency tympanometric results.
\end{abstract}

\section{INTRODUCTION}

Impedance audiometry has two main components: tympanometry and stapedius reflex measurement (1). Tympanometry is one of the most frequently performed and important components of the basic audiological evaluation. Objective middle ear assessment can best be performed by tympanometry (2). It measures how the middle-ear system responds to sound energy and changes in atmospheric pressure. It provides an objective, physiological measure of the acoustic admittance of the middle ear with the aid of air pressure in a sealed ear canal (3). The permeability and resistance of the middle ear system is defined with tympanometry via measuring the response to a sound stimulus using a microphone while pressure is changed along the external ear canal (4). The purpose of tympanometry is to asses audition on the basis of an objective description of the integrity and compliance of the eardrum, the mobility of the ossicular chain, the function of middle ear muscles and their attachments, and the size of the external ear canal (3). Data can be obtained from a tympanometer are estimated volume of air in front of the probe (equivalent ear canal volume, $\mathrm{V}_{\mathrm{e}}$ ), maximum compliance $\left(\mathrm{Y}_{\mathrm{tm}}\right)$, pressure at which the middle ear system has the greatest absorption of sound energy (TPP) and gradient of the tympanogram curve (5).

Qualitative and quantitative approaches have been used in the interpretation of 226-Hz tympanograms (6). Tympanograms can most simply be classified as types $\mathrm{A}, \mathrm{B}$, and $\mathrm{C}$ depending on the curve shape compared to 0 as the pressure is changed (2). Further classification according to the shape of the curve is also used widely, type $A$ as normal and type $B, C, A_{D}$ and $A_{s}$ as abnormal. Quantitative analysis of tympanograms is prefered, especially when evaluating infants and children, for whom different age-based normative values are needed (6). Definitions within modified Jerger classifications of tympanogram is described according to middle ear compliance $\left(\mathrm{Y}_{\mathrm{tm}}\right.$ in $\mathrm{ml}$ ) and middle ear pressure (TPP in daPa) as: type $\mathrm{A}$ $\left(Y_{t m}^{t m} \geq 0.2\right.$; TPP above -100$), C 1\left(Y_{t m} \geq 0.2\right.$; TPP below or equal to -100 and above -200$), \mathrm{C} 2\left(\mathrm{Y}_{\mathrm{tm}} \geq 0.2\right.$; TPP below or equal to -100 and above -400$)$ and $B\left(Y_{t m}<0.2\right.$; TPP below or equal to -400). Clinicians usually combine $\mathrm{A}$ and $\mathrm{C} 1$ as normal (7).

The aim of this study was to evaluate the usefulness and consistency of the $226 \mathrm{~Hz}$ tympanometer and its values in healthy cats. However, there are early tympanometry articles with cats, according to the authors knowledge these are the first published in vivo normal tympanometry values.

\section{MATERIAL and METHODS}

Cats brought to Mehmet Akif Ersoy University Animal Hospital for elective surgery without ear problems were included in the study. The study was approved by the Ethics Commission of Mehmet Akif Ersoy University. Fifteen mixed breed male (n: 7) and female (n: 8) cats at the age between 12-24 months included in the study. All cats were clinically 
healthy with no history of ear disease classified as ASA-I were anesthetized with xylazine $\mathrm{HCl} 1 \mathrm{mg} / \mathrm{kg}$ IM (20 mg / ml) (Alfazyne \%2, Alfasan, Woerden, Netherlands) - ketamine $\mathrm{HCl} 11 \mathrm{mg} / \mathrm{kg} \mathrm{IM}(100 \mathrm{mg} / \mathrm{ml}$ ) (Alfamine \%10, Alfasan, Woerden, Netherlands). 20 minutes after induction of the anaesthesia video-otoscopy used to examine external ear canal and tympanic membrane (Karl Storz, Telecam 20212030 PAL model, ABD) with an attached camera (Karl Storz, Tele Pack Vet X, ABD) and screen. All external ear canal and tympanic membrane images were obtained from both ears (Figure 1). Just after recording video-otoscopic healthy ear images, airtight seal obtained using $13 \mathrm{~mm}$ mushroom shaped rubber probe tips (Figure 2a). Tympanograms recorded from both ears at right and left lateral recumbency. Tympanometric measurements performed (Interacoustics AT235h $\AA$, Danmark) in both ears at $226 \mathrm{~Hz}$ probe tone frequency (Figure $2 \mathrm{~b}$ ). Air pressure -300 to $+300 \mathrm{~mm} \mathrm{H}_{2} \mathrm{O}$ was introduced by the probe to elicit the tympanograms.

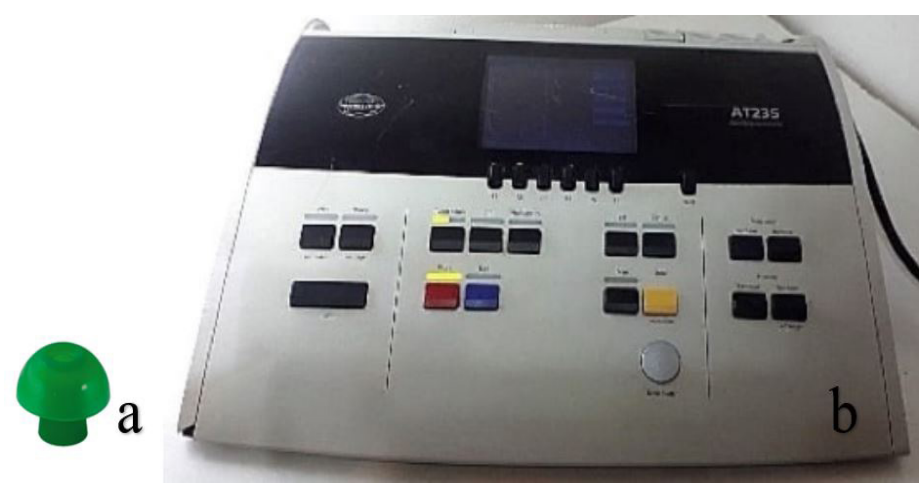

Figure 2. $13 \mathrm{~mm}$ mushroom shaped rubber probe tips (a) and audiometry device (Interacoustics AT235h®, Danmark) (b).

\section{RESULTS}

The study consisted of 15 domestic shorthaired cats (7 male, 8 female) ages between 12-24 months. Video-otoscopic examination confirmed the absence of inflammation or any other obvious explanation for the noncompliant cats. 30 healthy ears evaluated according to equivalent ear canal volume $\left(\mathrm{V}_{\mathrm{ea}}\right)$, static acoustic admittance $\left[\mathrm{Y}_{\mathrm{tm}}\right.$ (compliance)], tympanometric peak pressure (TPP) and gradient values under general anesthesia (Figure 3). Tympanometric measurements obtained from right and left ears were $0.61 \pm 0.12 \mathrm{ml}$ and 0.60 $\pm 0.12 \mathrm{ml} \mathrm{V}_{\mathrm{ea}}, 0.31 \pm 0.11 \mathrm{ml}$ and $0.31 \pm 0.11 \mathrm{ml} \mathrm{Y}_{\mathrm{tm}},-11.2$ $\pm 26.9 \mathrm{daPa}$ and $-14.9 \pm 29.2 \mathrm{daPa}$ TPP, $0.19 \pm 0.11 \mathrm{ml}$ and $0.24 \pm 0.18 \mathrm{ml}$ gradient respectively. Student's t test was used to evaluate the significance of the differences. There was no significant difference $(\mathrm{P}>0.1)$ between any values for right versus left (Table 1).

All tympanograms were classified as type A $\left(\mathrm{Y}_{\mathrm{tm}} \geq 0.2\right.$; TPP above -100) according to modified Jerger (2) classification scheme. One or two attempts required to obtain airtight seal with the choosen ear probe tip to get results from the tympanometric measurements.

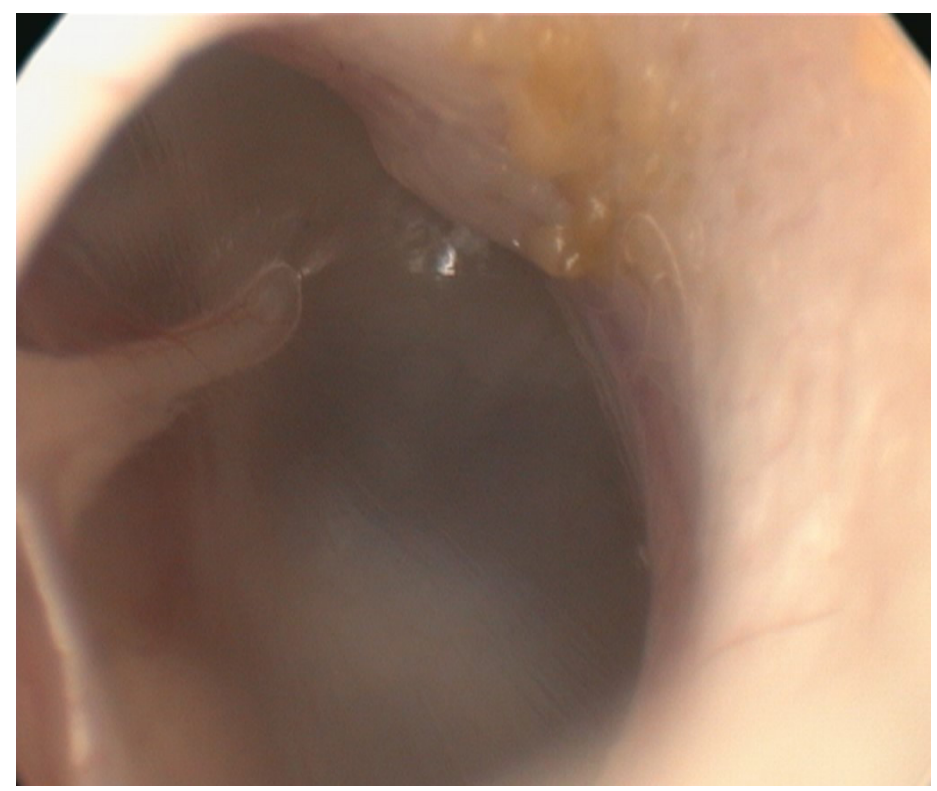

Figure 1. Video-otoscopic healthy external ear canal and tympanic membrane images obtained from cats

\section{DISCUSSION}

Before performing tympanometry, the audiologist should examine the ear canal with otoscopy for cerumen blockages, foreign bodies, drainage, TM perforation, or collapsed ear canal by otoscopy. Any of these conditions can affect estimates of ear canal volume and other tympanometry measurements and thus should be documented (6). The lighting and magnification are enhanced with video-otoscopy (8). Videootoscopy is an important step that should not be skipped before tympanometry. By the aid of video-otoscopy healthy external ear canal and tympanic membrane images was easily found.

The Vea, is affected by the position of the probe tip, the dimensions of the ear canal, and the amount of volume occupied by cerumen (6). Tympanometry objectively measures tympanic membrane mobility, middle ear function and ear canal volume which is normally $0.3-0.9 \mathrm{ml}$ in children (9). A normal tympanogram has a single clearly defined peak occurring near atmospheric pressure, type A. Problems in the middle ear cause

Table 1. Fifteen cats tympanometric Vea, Ytm, TPP and gradient measurement results from both ears (Two-Sample T-Test)

\begin{tabular}{lccc}
\hline & $\mathrm{n}: 15$ & Average \pm SD & P-value \\
\hline $\mathbf{V}_{\mathrm{ea}}(\mathbf{m l})$ & $\mathrm{R}$ & $0,61 \pm 0,12$ & 0.87 \\
& $\mathrm{~L}$ & $0,60 \pm 0,12$ & \\
$\mathbf{Y}_{\mathrm{tm}}(\mathrm{ml})$ & $\mathrm{R}$ & $0,31 \pm 0,11$ & 0.92 \\
& $\mathrm{~L}$ & $0,31 \pm 0,11$ & \\
$\mathbf{T P P}(\mathbf{d a P a})$ & $\mathrm{R}$ & $-11,2 \pm 26,9$ & \multirow{2}{*}{0.72} \\
& $\mathrm{~L}$ & $-14,9 \pm 29,2$ & \\
Gradient (ml) & $\mathrm{R}$ & $0,19 \pm 0,11$ & 0.38 \\
& $\mathrm{~L}$ & $0,24 \pm 0,18$ & \\
\hline
\end{tabular}

R: right, L: left, SD: Standart deviation

$\mathrm{P}>0.05$ there was no statistical difference 

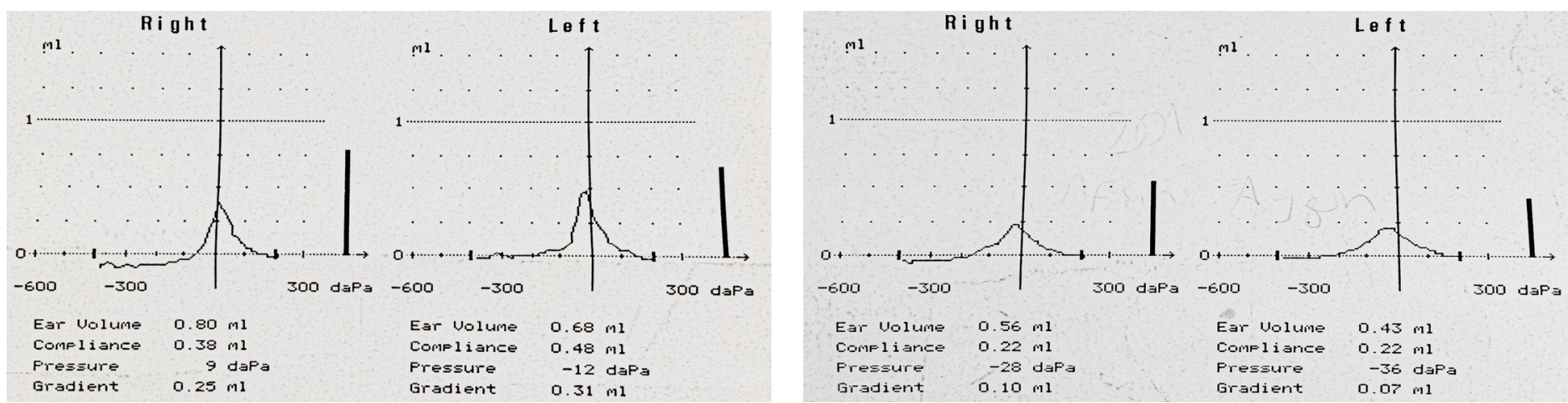

Figure 3. Tympanograms of two different cats with ear canal volume, compliance, pressure and gradient measurements

alterations in the shape of the tympanogram. For example, the most common pathology that affects tympanometry is fluid in the middle ear space, or otitis media with effusion (3). A normal ear canal volume $\left(\mathrm{V}_{\mathrm{e}}\right)$ of a cat found like the children. Hence the less anatomic variance in cats and narrow age range (12-24 months) in this study, obtained similar tympanometry measurements can be a good indicator distinguishing normal and abnormal situations in cats.

Halothane has been shown to increase compliance in dogs (10). In a study (11) cats were anesthetized with xylazine and ketamine to perform tympanometry. Although the results were not mentioned, this anaesthesia protocol was suggested to preserve compliance (12). All cases were anesthetized with xylazine and ketamine in this study prevented any anaesthesia related changes in tympanometry values.

Often it was necessary to support the probe assembly with the hand. However, the proximity and consistency of the results suggested that tympanometry is an objective measurement method that can be used in cats.

Normative ranges of tympanometric measurements of cats have not been published before according to authors knowledge. In a study (13), tympanogram morphologic findings in anesthetized cats differed between ascending and descending pressure directions but tympanometric measurements were not mentioned. Another experimental study measuring the acoustic impedance at the eardrum at constant sound pressure level in anesthetized cats and rabbits (14) did not unveil these tympanometric values. Since limited cases examined in this study, more needed to delimitate normative ranges and distinguish abnormalities for exact diagnosis.

\section{CONCLUSION}

In summary, audiometric tympanometry offers a simple, sensitive, and objective diagnostic method that can aid the veterinarian in the diagnosis and management of middle-ear disease. Less than one minute per ear required to complete the test is another advantage. Other diagnostic clues could be obtained with experiencing normative tympanograms like wideband and multi frequency in cats.

\section{CONFLICT of INTEREST}

The authors declare no conflicts of interest with respect to the publication of this manuscript.

\section{REFERENCES}

1. Moller AR. An Experimental Study of the Acoustic Impedance of the Middle Ear and Its Transmission Properties. Acta Otolaryngol. 1965; 60:129-149.

2. Jerger J. Clinical experience with impedance audiometry. Arch Otolaryngol. 1970; 92(4):311-324.

3. Sims MH. Clinical Evaluation of Auditory Function. In: Slatter D, editor. Textbook of Small Animal Surgery 3rd edn. Philadelphia, PA, USA: Elsevier Saunders; 2002. p. 1767-1773.

4. Harlor AD Jr, Bower C. Hearing assessment in infants and children: recommendations beyond neonatal screening. Pediatrics. 2009; 124(4):1252-1263.

5. Onusko E. Tympanometry. American Family Physician. 2004; 70 (9): 1713 - 1720.

6. Hunter LL, Sanford CA. Typmanometry and Wideband Acoustic Immitance. In: Marshall Chasin, Kristina English, Linda J. Hood, Kim L, editor(s). Handbook of Clinical Audiology. 7th ed. DanmarkTillery; 2015. p.137-140.

7. Haggard, MP., Higson, JM., Spencer, H. Air-conduction estimated from tympanometry (ACET) 1: Relationship to measured hearing in OME. Int J Pediatr Otorhinolaryngol. 2009 Jan;73(1):21-42.

8. Fiellau-Nikolajsen M. Tympanometry and secretory otitis media. Observations on diagnosis, epidemiology, treatment, and prevention in prospective cohort studies of three-year-old children. Acta Otolaryngol Suppl. 1983 Jul;394:1-73.

9. Schilder AG, Chonmaitree T, Cripps AW, et al. Otitis media. Nat Rev Dis Primers. 2016 Sep;2:160-63.

10. Kitzman JV, Chambers JN, Coulter DB. The effects of halothane-and-oxygen anesthesia, and of halothane-nitrous oxide-and-oxygen anesthesia on tympanograms in the dog. J Aud Res. 1982;22(2):87-92.

11. Sims MH. Electrodiagnostic evaluation of auditory function. Vet Clin North Am Small Anim Pract. 1988; 


\section{8(4):913-44.}

12. Strain GM, Fernandes AJ. Handheld tympanometer measurements in conscious dogs for the evaluation of the middle ear and auditory tube. Vet Dermatol. 2015; 26(3):193197.

13. Osguthorpe JD, Lam C. Methodologic aspects of tympanometry in cats. Otolaryngol Head Neck Surg. 1981; 89(6):1037-1040.

14. Aage R. Møller. An Experimental Study of the Acoustic Impedance of the Middle Ear and Its Transmission Properties, Acta Oto-Laryngologica. 1965; 60:1-6, 129-149. 